\title{
Closed form solutions for symmetric water filling games
}

\author{
E. Altman \\ INRIA Sophia Antipolis, France \\ Email: altman@sophia.inria.fr
}

\author{
K. Avrachenkov \\ INRIA Sophia Antipolis, France \\ Email: k.avrachenkov@sophia.inria.fr
}

\author{
A. Garnaev \\ St. Petersburg State University, Russia \\ Email: agarnaev@ rambler.ru
}

\begin{abstract}
We study power control in optimization and game frameworks. In the optimization framework there is a single decision maker who assigns network resources and in the game framework users share the network resources according to Nash equilibrium. The solution of these problems is based on so-called water-filling technique, which in turn uses bisection method for solution of non-linear equations for Lagrange multipliers. Here we provide a closed form solution to the water-filling problem, which allows us to solve it in a finite number of operations. Also, we produce a closed form solution for the Nash equilibrium in symmetric Gaussian interference game with an arbitrary number of users. Even though the game is symmetric, there is an intrinsic hierarchical structure induced by the quantity of the resources available to the users. We use this hierarchical structure to perform a successive reduction of the game. In addition to its mathematical beauty, the explicit solution allows one to study limiting cases when the crosstalk coefficient is either small or large. We provide an alternative simple proof of the convergence of the Iterative Water Filling Algorithm. Furthermore, it turns out that the convergence of Iterative Water Filling Algorithm slows down when the crosstalk coefficient is large. Using the closed form solution, we can avoid this problem. Finally, we compare the non-cooperative approach with the cooperative approach and show that the non-cooperative approach results in a more fair resource distribution.
\end{abstract}

\section{INTRODUCTION}

In wireless networks and DSL access networks the total available power for signal transmission has to be distributed among several resources. In the context of wireless networks, the resources may correspond to frequency bands (e.g. as in OFDM), or they may correspond to capacity available at different time slots. In the context of DSL access networks, the resources correspond to available frequency tones. This spectrum of problems can be considered in either optimization scenario or game scenario. The optimization scenario leads to "Water Filling Optimization Problem" [3], [6], [14] and the game scenario leads to "Water Filling Game" or "Gaussian Interference Game" [8], [11], [12], [15]. In the optimization scenario, one needs to maximize a concave function (Shannon capacity) subject to power constraints. The Lagrange multiplier corresponding to the power constraint is determined by a non-linear equation. In the previous works [3], [6], [14], it was suggested to find the Lagrange multiplier by means of a bisection algorithm, where comes the name "Water Filling Problem". Here we show that the Lagrange

\footnotetext{
${ }^{1}$ The work was partly supported by RFBR and NNSF Grant no.06-01-39005
}

multiplier and hence the optimal solution of the water filling problem can be found in explicit form with a finite number of operations. In the multiuser context, one can view the problem in either cooperative or non-cooperative setting. If a centralized controller wants to maximize the sum of all users' rates, the controller will face a non-convex optimization problem with many local maxima [13]. On the other hand, in the non-cooperative setting, the power allocation problem becomes a game problem where each user perceives the signals of the other users as interference and maximizes a concave function of the noise to interference ratio. A natural approach in the non-cooperative setting is the application of the Iterative Water Filling Algorithm (IWFA) [16]. Recently, the authors of [10] proved the convergence of IWFA under fairly general conditions. In the present work we study the case of symmetric water filling game. There is an intrinsic hierarchical structure induced by the quantity of the resources available to the users. We use this hierarchical structure to perform a successive reduction of the game, which allows us to find Nash equilibrium in explicit form. In addition, to its mathematical beauty, the explicit solution allows one to find the Nash equilibrium in water filling game in a finite number of operations and to study limiting cases when the crosstalk coefficient is either small or large. As a by-product, we obtain an alternative simple proof of the convergence of the Iterative Water Filling Algorithm. Furthermore, it turns out that the convergence of IWFA slows down when the crosstalk coefficient is large. Using the closed form solution, we can avoid this problem. Finally, we compare the non-cooperative approach with the cooperative approach and conclude that the cost of anarchy is small in the case of small crosstalk coefficients and that the decentralized solution is better than the centralized one with respect to fairness. Applications that can mostly benefit from decentralized non-cooperative power control are ad-hoc and sensor networks with no predefined base stations [4], [9], [7]. An interested reader can find more references on non-cooperative power control in [2], [8]. We would like to mention that the water filling problem and jamming games with transmission costs have been analyzed in [1].

The paper is organized as follows: In Section II we recall the single decision maker setup of the water filling optimization problem and provide its explicit solution. Then in Sections IIIVII we formulate multiuser symmetric water filling game and 
characterize its Nash equilibrium, also we give an alternative simple proof of the convergence of the iterative water filling algorithm and suggest the explicit form of the users' strategy in the Nash equilibrium. In Section VIII we confirm our finding with the help of numerical examples and compare the decentralized approach with the centralized one.

\section{SINGLE DECISION MAKER}

First let us consider the power allocation problem in the case of a single decision maker. The single decision maker (also called "user" or "transmitter") wants to send information using $n$ independent resources so that to maximize the Shannon capacity. We further assume that resource $i$ has a "weight" of $\pi_{i}$.

Possible interpretations:

(i) The resources may correspond to capacity available at different time slots; we assume that there is a varying environment whose state changes among a finite set of states $i \in[1, n]$, according to some ergodic stochastic process with stationary distribution $\left\{\pi_{i}\right\}_{i=1}^{n}$. We assume that the user has perfect knowledge of the environment state at the beginning of each time slot.

(ii) The resources may correspond to frequency bands (e.g. as in OFDM) where one should assign different power levels for different sub-carriers [14]. In that case we may take $\pi_{i}=1 / n$ for all $i$.

The strategy of user is $T=\left(T_{1}, \ldots, T_{n}\right)$ with $\sum_{i=1}^{n} \pi_{i} T_{i}=$ $\bar{T}, T_{i} \geq 0, \pi_{i}>0$ for $i \in[1, n]$ and $\bar{T}>0$. As the payoff to user we take the Shannon capacity

$$
v(T)=\sum_{i=1}^{n} \pi_{i} \ln \left(1+T_{i} / N_{i}^{0}\right),
$$

where $N_{i}^{0}>0$ is the noise level in the sub-carrier $i$.

We would like to emphasize that this generalized description of the water-filling problem can be used for power allocation in time as well as power allocation in space-frequency. Following the standard water-filling approach [3], [6], [14] we have the following result.

Theorem 1: Let $T_{i}(\omega)=\left[1 / \omega-N_{i}^{0}\right]_{+}$for $i \in[1, n]$ and $H(\omega)=\sum_{i=1}^{n} \pi_{i} T_{i}(\omega)$. Then $T\left(\omega^{*}\right)=\left(T_{1}\left(\omega^{*}\right), \ldots, T_{n}\left(\omega^{*}\right)\right)$ is the unique optimal strategy and its payoff is $v\left(T\left(\omega^{*}\right)\right)$ where $\omega^{*}$ is the unique root of the equation

$$
H(\omega)=\bar{T} \text {. }
$$

In the previous studies of the water-filling problems it was suggested to use numerical (e.g., bisection) method to solve the equation (1). Here we propose an explicit form approach for its solution.

Without loss of generality we can assume that by the noise levels the sub-carriers are arranged in decreasing order:

$$
N_{n}^{0} \geq N_{n-1}^{0} \geq \ldots \geq N_{1}^{0} .
$$

Then, since $H(\cdot)$ is decreasing, we have the following result:
Theorem 2: The solution of the water-filling optimization problem is given by

$$
T_{i}^{*}= \begin{cases}\left(\bar{T}+\sum_{t=1}^{k} \pi_{t}\left(N_{t}^{0}-N_{i}^{0}\right)\right) /\left(\sum_{t=1}^{k} \pi_{t}\right), & i \leq k, \\ 0, & i>k\end{cases}
$$

where $k$ can be found from the following condition:

$$
\varphi_{k}<\bar{T} \leq \varphi_{k+1}
$$

where

$$
\varphi_{t}=\sum_{i=1}^{t} \pi_{i}\left(N_{t}^{0}-N_{i}^{0}\right) \text { for } t \in[1, n] \text { and } \varphi_{n+1}=\infty .
$$

Thus, contrary to the numerical (bisection) approach, in order to find an optimal resource allocation we need to execute only a finite number of operations.

\section{SyMMETRIC WATER FILLING GAME}

Let us now consider a multi-user scenario. Specifically, we consider $L$ users who try to send information through $n$ resources so that to maximize their transmission rates. The strategy of user $j$ is $T^{j}=\left(T_{1}^{j}, \ldots, T_{n}^{j}\right)$ subject to

$$
\sum_{i=1}^{n} \pi_{i} T_{i}^{j}=\bar{T}^{j}
$$

where $\bar{T}^{j}>0$ for $j \in[1, L]$. The element $T_{i}^{j}$ is the power level used by transmitter $j$ when the environment is in state $i$. The payoff to user $j$ is given as follows:

$$
v^{j}\left(T^{1}, \ldots, T^{L}\right)=\sum_{i=1}^{n} \pi_{i} \ln \left(1+\frac{\alpha_{i}^{j} T_{i}^{j}}{N_{i}^{0}+g_{i} \sum_{k \neq j} \alpha_{i}^{k} T_{i}^{k}}\right),
$$

where $N_{i}^{0}$ is the noise level and $g_{i} \in(0,1)$ and $\alpha_{i}^{j}$ are fading channel gains of user $j$ when the environment is in state $i$. These payoffs correspond to Shannon capacities. The constraint (3) corresponds to the average power consumption constraint. This is an instance of the Water Filling or Gaussian Interference Game [8], [11], [12], [15], [16]. In the important particular cases of OFDM wireless network and DSL access network, $\pi_{i}=1 / n, i=1, \ldots, n$.

We will look for a Nash Equilibrium (NE) of this problem. The strategies $T^{1 *}, \ldots, T^{L *}$ constitute a NE, if for any strategies $T^{1}, \ldots, T^{L}$ the following inequalities hold:

$$
\begin{aligned}
v^{1}\left(T^{1}, T^{2 *}, \ldots, T^{L *}\right) & \leq v^{1}\left(T^{1 *}, T^{2 *} \ldots, T^{L *}\right), \\
& \ldots \\
v^{L}\left(T^{1 *}, \ldots, T^{(L-1) *}, T^{L}\right) & \leq v^{L}\left(T^{1 *}, \ldots, T^{(L-1) *}, T^{L *}\right) .
\end{aligned}
$$

To find NE of such game usually the following numerical algorithm is applied. First, a strategy of $L-1$ users (say, user $2, \ldots, L)$ are fixed. Then, the best reply of user 1 is found solving the Water Filling optimization problem. Then, the best reply of user 2 on these strategies of the users is found solving the optimization problem and so on. It is possible to prove that under some assumption on fading channel gains this sequence of the strategies converge to a NE [10]. 
In this work we restrict ourselves to the case of symmetric game with equal crosstalk coefficients. This situation can for example correspond to the scenario when the users are situated at about the same distance from the base station. Namely, we assume that $\alpha_{i}^{1}=\ldots=\alpha_{i}^{L}$ and $g_{i}=g$ for $i \in(0,1)$. So, in our case the payoffs to users are given as follows

$$
v^{j}\left(T^{1}, \ldots, T^{L}\right)=\sum_{i=1}^{n} \pi_{i} \ln \left(1+\frac{T_{i}^{j}}{N_{i}^{0}+g \sum_{k \neq j} T_{i}^{k}}\right),
$$

where $N_{i}^{0}:=N_{i}^{0} / \alpha_{i}, i \in[1, n]$ and without loss of generality we can assume that the sub-carriers are arranged by the noiselevels are arranged in decreasing order (2). We would like to emphasize that the dependance of $N_{i}^{0}$ on $i$ allows us to model an environment with varying transmission conditions.

For this problem we propose a new algorithm of finding the NE. The algorithm is based on closed form expressions and hence it requires only a finite number of operations. Also, explaining this algorithm we will prove that the game has the unique NE under assumption that $g \in(0,1)$.

Since $v_{j}$ is concave on $T^{j}$, the Kuhn-Tucker Theorem implies the following theorem.

Theorem 3: $\left(T^{1 *}, \ldots, T^{L *}\right)$ is a Nash equilibrium if and only if there are non-negative $\omega^{j}, j \in[1, L]$ (Lagrange multipliers) such that

$$
\begin{aligned}
& \frac{\partial}{\partial T_{i}^{j}} v^{j}\left(T^{1 *}, \ldots, T^{L *}\right)=\frac{1}{T_{i}^{j *}+N_{i}^{0}+g \sum_{k \neq j} T_{i}^{k *}} \\
& \begin{cases}=\omega^{j} & \text { for } T_{i}^{j *}>0, \\
\leq \omega^{j} & \text { for } T_{i}^{j *}=0 .\end{cases}
\end{aligned}
$$

It is clear that all $\omega^{j}$ are positive.

The assumption that $g<1$ is crucial for uniqueness of equilibrium as it is shown in the following proposition.

Proposition 1: For $g=1$ the symmetric water filling game has infinite number (continuum) of Nash equilibria.

Proof. Suppose that $\left(T^{1 *}, \ldots, T^{L *}\right)$ is a Nash equilibrium. Then, by Theorem 3, there are non-negative $\omega^{j}, j \in[1, L]$ such that

$$
1 /\left(N_{i}^{0}+\sum_{k=1}^{L} T_{i}^{k *}\right) \begin{cases}=\omega^{j} & \text { for } T_{i}^{j *}>0 \\ \leq \omega^{j} & \text { for } T_{i}^{j *}=0\end{cases}
$$

Thus, $\omega^{1}=\ldots=\omega^{L}=\omega$. So, $T_{i}^{1 *}, \ldots, T_{i}^{L *}, i \in[1, n]$ have to be any non-negative such that

$$
\sum_{k=1}^{L} T_{i}^{k *}=\left[1 / \omega-N_{i}^{0}\right]_{+},
$$

and

$$
\sum_{i=1}^{n} \pi_{i} T_{i}^{k *}=\bar{T}^{k} \text { for } k \in[1, L],
$$

where $\omega$ is the unique positive root of the equation

$$
\sum_{i=1}^{n} \pi_{i}\left[1 / \omega-N_{i}^{0}\right]_{+}=\sum_{k=1}^{L} \bar{T}^{k}
$$

It is clear that there are infinite number of such strategies. For example, if $T_{i}^{a *}$ and $T_{i}^{b *}, i \in[1, n](a \neq b)$ is the one of them and $T_{k}^{a *}>0$ and $T_{m}^{b *}>$ for some $k \neq m$. Then, it is clear that the following strategies for any small enough positive $\epsilon$ are also optimal:

$$
\begin{aligned}
& \tilde{T}_{i}^{a *}= \begin{cases}T_{i}^{a *} & \text { for } i \neq k, m, \\
T_{i}^{a *}+\epsilon & \text { for } i=k, \\
T_{i}^{a *}-\epsilon \pi_{k} / \pi_{m} & \text { for } i=m,\end{cases} \\
& \tilde{T}_{i}^{b *}= \begin{cases}T_{i}^{b *} & \text { for } i \neq k, m, \\
T_{i}^{b *}-\epsilon & \text { for } i=k, \\
T_{i}^{b *}+\epsilon \pi_{k} / \pi_{m} & \text { for } i=m .\end{cases}
\end{aligned}
$$

This completes the proof of Proposition 1.

\section{A RECURSIVE APPROACH TO THE SYMMETRIC WATER FILLING GAME}

Let $\omega^{1}, \ldots, \omega^{L}$ be some parameters which in the future will act as Lagrangian multipliers. Using these parameters we introduce some auxiliary notations. Assume that these parameters are arranged as follows (this assumption does not reduce the generality of our forthcoming conclusions):

$$
\omega^{1} \leq \ldots \leq \omega^{L} .
$$

Also denote

$$
\bar{\omega}=\left(\omega^{1}, \ldots, \omega^{L}\right) .
$$

Introduce the following auxiliary sequence:

$$
t^{r}=\frac{1}{1-g}\left(\frac{1+(r-1) g}{\omega^{r}}-g \sum_{j=1}^{r} \frac{1}{\omega^{j}}\right) \text { for } r \in[1, L] .
$$

It is clear that by (5)

$$
t^{r+1}=\frac{1+(r-1) g}{1-g}\left(\frac{1}{\omega^{r+1}}-\frac{1}{\omega^{r}}\right)+t^{r} \leq t^{r} .
$$

Thus,

$$
t^{L} \leq t^{L-1} \leq \ldots \leq t^{1}
$$

and

$$
\frac{1}{\omega^{r+1}}-\frac{1}{\omega^{r}}=\frac{1-g}{1+(r-1) g}\left(t^{r+1}-t^{r}\right) .
$$

Hence, for $j \in[k+1, L]$ we have:

$$
\frac{1}{\omega^{k}}-\frac{1}{\omega^{j}}=\sum_{r=k}^{j-1} \frac{1-g}{1+(r-1) g}\left(t^{r}-t^{r+1}\right) .
$$

Then, sequences $\left\{\omega^{r}\right\}$ and $\left\{t^{r}\right\}$ has the following recurrent relations:

$$
\begin{aligned}
\frac{1}{\omega^{1}} & =t^{1}, \quad \frac{1}{\omega^{2}}=(1-g) t^{2}+g t^{1}, \\
\frac{1}{\omega^{r+1}} & =\frac{1-g}{1+(r-1) g} t^{r+1} \\
& +\sum_{j=2}^{r} \frac{(1-g) g}{(1+(j-1) g)(1+(j-2) g)} t^{j}+g t^{1},
\end{aligned}
$$


where $r \geq 1$. If we know the sequence $\left\{t^{r}\right\}$ we can restore the sequence $\left\{\omega^{r}\right\}$. Thus, these two sequences are equivalent.

Introduce one more auxiliary sequence as follows:

$$
\tau_{r}^{k}=\frac{1}{1-g}\left(\frac{1+(L-1-r+k) g}{\omega^{k}}-g \sum_{j=1}^{L-r+k} \frac{1}{\omega^{j}}\right),
$$

where $r \in[k, L], k \in[1, L]$. There is a simple relation between sequences $\left\{\omega^{k}\right\},\left\{t^{k}\right\}$ and $\left\{\tau_{r}^{k}\right\}$ :

$$
\tau_{L}^{k}=t^{k}
$$

and

$$
\tau_{r}^{k}=\frac{1+(L-1-r+k) g}{1-g}\left(\frac{1}{\omega^{k}}-\frac{1}{\omega^{L-r+k}}\right)+t^{L-r+k} .
$$

So, by (7), collecting terms which depend on $t^{k}$ we obtain

$$
\tau_{r}^{k}=b^{k, r} t^{k}+A^{k, r}
$$

where

$$
b^{k, r}=\frac{1+(L-1-r+k) g}{1+(k-1) g},
$$

and

$$
\begin{aligned}
A^{k, r} & =-g \sum_{j=k+1}^{L-r+k-1} \frac{1+(L-1-r+k) g}{(1+(j-1) g)(1+(j-2) g)} t^{j} \\
& -\frac{g}{(1+(L-2-r+k) g} t^{L-r+k} .
\end{aligned}
$$

Thus, $A^{k, r}$ depends only on $\left\{t^{j}\right\}$ with $j>k$.

Finally introduce the following notation:

(a) for $N_{i}^{0}<t^{L}$

$$
T_{i}^{k}(\bar{\omega})=\frac{1}{1+(L-1) g}\left(\tau_{k}^{k}-N_{i}^{0}\right),
$$

(b) $t^{L+k+1-r} \leq N_{i}^{0}<t^{L+k-r}$ where $r \in[k+1, L]$

$$
T_{i}^{k}(\bar{\omega})=\frac{1}{1+(L-1-r+k) g}\left(\tau_{r}^{k}-N_{i}^{0}\right),
$$

(c) for $t^{k} \leq N_{i}^{0}$

$$
T_{i}^{k}(\bar{\omega})=0
$$

For other combinations of relations between $\omega^{j}, j \in[1, L]$, $T_{i}^{k}$ are defined by symmetry. By Theorem 3 we have the following result.

Theorem 4: Each Nash equilibrium is of the form $\left(T^{1}(\bar{\omega}), \ldots, T^{L}(\bar{\omega})\right)$.

The next lemma provides a nice relation between $L$ and $L-1$ person games which shows that the introduction of a new user into the game leads to a bigger competition for the better quality channels meanwhile users prefer to keep the old structure of their strategies for worse quality channels.

Lemma 1: Let $\left(T^{1, L}\left(\omega^{1}, \ldots, \omega^{L}\right), \ldots, T^{L, L}\left(\omega^{1}, \ldots, \omega^{L}\right)\right)$ given by Theorem 4 (here we added the second super-script index in the notation of the strategies in order to emphasize that the strategies depend on the number of users). Then, we have

$$
T_{i}^{k, L}\left(\omega^{1}, \ldots, \omega^{L}\right)= \begin{cases}\frac{\tau_{k}^{k}-N_{i}^{0}}{1+(L-1) g} & \text { for } N_{i}^{0}<t^{L} \\ T_{i}^{k, L-1}\left(\omega^{1}, \ldots, \omega^{L-1}\right) & \text { for } t^{L} \leq N_{i}^{0}\end{cases}
$$

where $k \in[1, L-1]$ and

$$
T_{i}^{L, L}\left(\omega^{1}, \ldots, \omega^{L}\right)= \begin{cases}\frac{t^{L}-N_{i}^{0}}{1+(L-1) g} & \text { for } N_{i}^{0}<t^{L} \\ 0 & \text { for } t^{L} \leq N_{i}^{0}\end{cases}
$$

\section{A WATER-FILling ALGORITHM}

In this section we describe a version of the water-filling algorithm for finding the NE and supply a simple proof of its convergence based on some monotonicity properties. Let

$$
H^{k}(\bar{\omega})=\sum_{i=1}^{n} \pi_{i} T_{i}^{k}(\bar{\omega}) \text { for } k \in[1, L] .
$$

To find a NE we have to find $\bar{\omega}$ such that

$$
H^{k}(\bar{\omega})=\bar{T}^{k} \text { for } k \in[1, L] .
$$

It is clear that $H^{k}(\bar{\omega})$ has the following properties, collected in the next Lemma, which follow directly from the explicit formulas of the NE.

Lemma 2: (i) $H^{k}(\bar{\omega})$ is nonnegative and continuous, (ii) $H^{k}(\bar{\omega})$ is decreasing on $\omega^{k}$, (iii) $H^{k}(\bar{\omega}) \rightarrow \infty$ for $\omega^{k} \rightarrow 0$, (iv) $H^{k}(\bar{\omega})=0$ for enough big $\omega^{k}$, say for $\omega^{k} \geq 1 / N_{1}^{0}$, (v) $H^{k}(\bar{\omega})$ is non-decreasing by $\omega^{j}$ where $j \neq k$.

This properties give a simple proof of the convergence of the following iterative water filling algorithm for finding the NE.

Let $\omega_{0}^{k}$ for all $k \in[1, L]$ be such that $H^{k}\left(\bar{\omega}_{0}\right)=0$, for example $\omega_{0}^{k}=1 / N_{1}^{0}$. Let $\omega_{1}^{k}=\omega_{0}^{k}$ for all $k \in[2, L]$ and define $\omega_{1}^{1}$ such that $H^{1}\left(\bar{\omega}_{1}\right)=\bar{T}^{1}$. Such $\omega_{1}^{1}$ exists by Lemma 2(i)-(iii). Then, by Lemma 2(i),(v) $H^{k}\left(\bar{\omega}_{0}\right)=0$ for $k \in[2, L]$. Let $\omega_{2}^{k}=\omega_{1}^{k}$ for all $k \neq 2$ and define $\omega_{2}^{2}$ such that $H^{2}\left(\bar{\omega}_{2}\right)=\bar{T}^{2}$. Then, by Lemma 2(i),(v) $H^{k}\left(\bar{\omega}_{0}\right)=0$ for $k>2$ and $H^{k}\left(\bar{\omega}_{0}\right) \leq \bar{T}^{k}$ for $k=1$ and so on. Let $\omega_{L}^{k}=\omega_{L-1}^{k}$ for all $k \neq L$ and define $\omega_{L}^{L}$ such that $H^{L}\left(\bar{\omega}_{L}\right)=\bar{T}^{L}$. Then, by Lemma 2(i),(v) $H^{k}\left(\bar{\omega}_{L}\right) \leq \bar{T}^{k}$ for $k \neq L$ and so on. So we have non-increasing positive sequence $\omega^{k}$. Thus, it converges to an $\bar{\omega}_{*}$ which produces a NE.

\section{EXISTENCE AND UNIQUENESS OF THE NASH EQUILIBRIUM}

In this section we will prove existence and uniqueness of the Nash equilibrium for $L$ person symmetric water-filling game. Our proof will have constructive character which allows us to produce an effective algorithm for finding the equilibrium strategies.

First note that there is a monotonous dependence between the resources the users can apply and Lagrange multipliers.

Lemma 3: Let $\left(T^{1}(\bar{\omega}), \ldots, T^{L}(\bar{\omega})\right)$ be a Nash equilibrium. If

$$
\bar{T}^{1} \geq \ldots \geq \bar{T}^{L}
$$


then (5) holds.

Note that the assumption (13) does not collude with (2) since (13) arranges the maximal power levels available to the users and (2) arranges the noise levels present in the sub-carriers.

Proof. The result immediately follows from the following monotonicity property implied by explicit formulas of the Nash equilibrium, namely, if $\omega^{i}<\omega^{j}$ then $H^{i}(\bar{\omega})>H^{j}(\bar{\omega})$.

Without loss of generality we can assume that (13) holds. Thus, by Lemma 3, (5) also holds.

Let $\bar{\omega}$ be the positive solution of (12). Then, by Lemma 3, the relation (5) holds. To find $\bar{\omega}$ we have to solve the system of non-linear equations (12). It is quite bulky system and it looks hard to solve. We will not solve it directly. What we will do we express $\omega^{1}, \ldots \omega^{L}$ by $t^{1}, \ldots, t^{L}$, substitute these expression into (12). The transformed system will have a triangular form, namely

$$
\begin{aligned}
& \tilde{H}^{L}\left(t^{L}\right)=\bar{T}^{L}, \\
& \tilde{H}^{L-1}\left(t^{L-1}, t^{L}\right)=\bar{T}^{L-1}, \\
& \cdots \\
& \tilde{H}^{1}\left(t^{1}, \ldots, t^{L-1}, t^{L}\right)=\bar{T}^{1} .
\end{aligned}
$$

The last system, because of monotonicity properties of $\tilde{H}^{k}$ on $t^{k}$, can be easily solved. Now we can move on to construction of $\tilde{H}^{L}\left(t^{L}\right), \ldots, \tilde{H}^{1}\left(t^{1}, \ldots, t^{L-1}, t^{L}\right)$. First we will construct $\tilde{H}^{L}\left(t^{L}\right)$ and find the optimal $t^{L}$. Note that,

$$
\begin{aligned}
H^{L}(\bar{\omega}) & =\sum_{N_{i}^{0}<t^{L}} \pi_{i} T_{i}^{L}(\bar{\omega})= \\
& =\frac{1}{1+(L-1) g} \sum_{N_{i}^{0}<t^{L}} \pi_{i}\left(\tau_{L}^{L}-N_{i}^{0}\right) \\
& =\frac{1}{1+(L-1) g} \sum_{N_{i}^{0}<t^{L}} \pi_{i}\left(t^{L}-N_{i}^{0}\right)=\tilde{H}^{L}\left(t^{L}\right) .
\end{aligned}
$$

It is clear that $\tilde{H}^{L}(\cdot)$ is continuous in $(0, \infty), \tilde{H}^{L}(\tau)=0$ for $\tau \leq N_{1}^{0}, \tilde{H}^{L}(+\infty)=+\infty$ and $\tilde{H}^{L}(\cdot)$ is strictly increasing in $\left(N_{1}^{0}, \infty\right)$. Then, there is the unique positive $t_{*}^{L}$ such that

$$
\tilde{H}^{L}\left(t_{*}^{L}\right)=\bar{T}^{L}
$$

Now we move on to construction of $\tilde{H}^{L-1}\left(t^{L-1}, t^{L}\right)$ and finding the optimal $t^{L-1}$. Note that $\tau_{L}^{L-1}=t^{L-1}$ and by (7) and (10), we have

$$
\begin{aligned}
& \tau_{L-1}^{L-1}=\tau_{L}^{L-1}+\frac{g}{1-g}\left(\frac{1}{\omega^{L-1}}-\frac{1}{\omega^{L}}\right) \\
& =t^{L-1}+\frac{g}{1+(L-2) g}\left(t^{L-1}-t^{L}\right) \\
& =\frac{1+(L-1) g}{1+(L-2) g} t^{L-1}-\frac{g}{1+(L-2) g} t^{L} .
\end{aligned}
$$

Thus,

$$
\begin{aligned}
& H^{L-1}(\bar{\omega})=\sum_{N_{i}^{0}<t^{L}} \pi_{i} T_{i}^{L-1}(\bar{\omega})+\sum_{t^{L} \leq N_{i}^{0}<t^{L-1}} \pi_{i} T_{i}^{L-1}(\bar{\omega})= \\
& =\frac{1}{1+(L-1) g} \sum_{N_{i}^{0}<t^{L}} \pi_{i}\left(\tau_{L-1}^{L-1}-N_{i}^{0}\right) \\
& +\frac{1}{1+(L-2) g} \sum_{t^{L} \leq N_{i}^{0}<t^{L-1}} \pi_{i}\left(\tau_{L}^{L-1}-N_{i}^{0}\right) \\
& =\frac{1}{1+(L-1) g} \sum_{N_{i}^{0}<t^{L}} \pi_{i}\left(\frac{1+(L-1) g}{1+(L-2) g} t^{L-1}\right. \\
& \left.-\frac{g}{1+(L-2) g} t^{L}-N_{i}^{0}\right) \\
& +\frac{1}{1+(L-2) g} \sum_{t^{L} \leq N_{i}^{0}<t^{L-1}} \pi_{i}\left(t^{L-1}-N_{i}^{0}\right) \\
& =\tilde{H}^{L-1}\left(t^{L-1}, t^{L}\right) .
\end{aligned}
$$

It is clear that $\tilde{H}^{L-1}\left(\cdot, t_{*}^{L}\right)$ is continuous and increasing in $\left(t_{*}^{L}, \infty\right), \tilde{H}^{L-1}\left(\infty, t_{*}^{L}\right)=+\infty$ and $\tilde{H}^{L-1}\left(t_{*}^{L}, t_{*}^{L}\right)=$ $\tilde{H}^{L}\left(t_{*}^{L}\right)=\bar{T}^{L} \leq \bar{T}^{L-1}$. So, there is the unique positive $t_{*}^{L-1}$ such that

$$
\tilde{H}^{L-1}\left(t_{*}^{L-1}, t_{*}^{L}\right)=\bar{T}^{L} .
$$

Next we construct $\tilde{H}^{k}\left(t^{k}, \ldots, t^{L-1}, t^{L}\right)$ and find the optimal $t^{k}$ where $k \in[1, L-2]$. By (9) and (9), we have

$$
\begin{aligned}
& H^{k}(\bar{\omega})=\sum_{N_{i}^{0}<t^{L}} \pi_{i} T_{i}^{k}+\sum_{r=k+1}^{L} \sum_{t^{L+k+1-r} \leq N_{i}^{0}<t^{L+k-r}} \pi_{i} T_{i}^{k} \\
& =\frac{1}{1+(L-1) g} \sum_{N_{i}^{0}<t^{L}} \pi_{i}\left(\tau_{L}^{k}-N_{i}^{0}\right) \\
& +\sum_{r=k+1}^{L} \sum_{t^{L+k+1-r} \leq N_{i}^{0}<t^{L+k-r}} \frac{\pi_{i}\left(\tau_{r}^{k}-N_{i}^{0}\right)}{1+(L-1-r+k) g} \\
& =\frac{1}{1+(L-1) g} \sum_{N_{i}^{0}<t^{L}} \pi_{i}\left(b^{k, k} t^{k}+A^{k, k}-N_{i}^{0}\right) \\
& +\sum_{r=k+1}^{L} \sum_{t^{L+k+1-r} \leq N_{i}^{0}<t^{L+k-r}} \frac{\pi_{i}\left(b^{k, r} t^{k}+A^{k, r}-N_{i}^{0}\right)}{1+(L-1-r+k) g} \\
& =\tilde{H}^{k}\left(t^{k}, t^{k+1}, \ldots, t^{L}\right) .
\end{aligned}
$$

It is clear that $\tilde{H}^{k}\left(\cdot, t_{*}^{k+1}, \ldots, t_{*}^{L}\right)$ is continuous and increasing in $\left(t_{*}^{k+1}, \infty\right), \tilde{H}^{k}\left(\infty, t_{*}^{k+1}, \ldots, t_{*}^{L}\right)=+\infty$ and by Lemma 1 $\tilde{H}^{k}\left(t_{*}^{k+1}, t_{*}^{k+1}, \ldots, t_{*}^{L}\right)=\tilde{H}^{k+1}\left(t_{*}^{k+1}, \ldots, t_{*}^{L}\right)=\bar{T}^{k+1} \leq$ $\bar{T}^{k}$. So, there is the unique positive $t_{*}^{k}$ such that

$$
\tilde{H}^{k}\left(t_{*}^{k}, t_{*}^{k+1}, \ldots, t_{*}^{L}\right)=\bar{T}^{k} .
$$

Thus, we have proved the following result:

Lemma 4: Solution of the system (12) is equivalent to solution of the triangular system (14). This system has the unique solution which can be found sequentially from $t^{L}$ down to $t^{1}$, applying either the bisection method or the explicit scheme. The optimal Lagrangian multipliers can be reconstructed from $\left\{t^{r}\right\}$ by (8). 
Finally, building upon Lemma 4, we have the following result:

Theorem 5: The symmetric water filling game has the unique Nash equilibrium $\left(T^{1}\left(\bar{\omega}_{*}\right), \ldots, T^{L}\left(\bar{\omega}_{*}\right)\right)$, where $\bar{\omega}_{*}$ is given by (8).

Note that although the payoffs have symmetric form, the equilibrium strategies, because of triangular form of system (14), have hierarchical structure induced by difference in power levels available to the users. Namely, the user who has to transmit with smaller average power consumption, in our case it is user $L$, acts first. He assigns his optimal strategies as if there is no other users at all but taking into account the total number of users and fading channels gains. Then, the turn to act is given to user $L-1$. He takes into account only the behavior of the user $L$ with smaller average power consumption than he has, the total number of users and fading channels gains and so on. The last user who constructs the equilibrium strategy is user 1 with the largest available power resource.

\section{ClOSED FORM SOLUTION FOR $L$ PERSON GAME}

In this section for the case of $L$ users we show how Theorem 5 and Lemma 4 can be used to construct NE in closed form.

Assume that $\bar{T}^{1}>\ldots>\bar{T}^{L}$. We will construct the equilibrium strategies $T^{L *}, \ldots, T^{1 *}$ sequentially.

Step for construction of $T^{L *}$. Since $\tilde{H}^{L}(\cdot)$ is strictly increasing we can find an integer $k_{L}$ such that

$$
\tilde{H}^{L}\left(N_{k^{L}}^{0}\right)<\bar{T}^{L} \leq \tilde{H}^{L}\left(N_{k^{L}+1}^{0}\right)
$$

or from the following equivalent conditions:

$$
\varphi_{k^{L}}^{L}<\bar{T}^{L} \leq \varphi_{k^{L}+1}^{L}
$$

where

$$
\varphi_{k}^{L}=\frac{1}{1+(L-1) g} \sum_{i=1}^{k} \pi_{i}\left(N_{k}^{0}-N_{i}^{0}\right)
$$

for $k \leq n$, and $\varphi_{n+1}^{L}=\infty$. Then, since $\tilde{H}^{L}\left(t_{*}^{L}\right)=\bar{T}^{L}$, we have that

$$
t_{*}^{L}=\frac{(1+(L-1) g) \bar{T}^{L}+\sum_{i=1}^{k_{L}} \pi_{i} N_{i}^{0}}{\sum_{i=1}^{k_{L}} \pi_{i}} .
$$

Thus, the equilibrium strategy of user $L$ is given as follows

$$
T_{i}^{L *}= \begin{cases}\frac{1}{1+(L-1) g}\left(t_{*}^{L}-N_{i}^{0}\right) & \text { if } i \in\left[1, k^{L}\right], \\ 0 & \text { if } i \in\left[k^{L}+1, n\right] .\end{cases}
$$

Step for construction of $T^{(L-1) *}$. Since $t_{*}^{L-1}$ is the root of the equation $\tilde{H}^{L-1}\left(\cdot, t_{*}^{L}\right)=\bar{T}^{L-1}$ there is $k^{L-1}$ such that $k^{L-1} \geq k^{L}$ and $N_{k^{L-1}+1}^{0} \geq t_{*}^{L-1}>N_{k^{L-1}}^{0}$. Thus,

$$
\begin{aligned}
t_{*}^{L-1} & =\left(\bar{T}^{L-1}+\frac{1}{1+(L-2) g} \sum_{i=k^{L}+1}^{k^{L-1}} \pi_{i} N_{i}^{0}\right. \\
& \left.+\frac{1}{1+(L-1) g} \sum_{i=1}^{k^{L}} \pi_{i}\left(\frac{g t_{L}^{*}}{1+(L-2) g}+N_{i}^{0}\right)\right) \\
& /\left(\frac{1}{1+(L-2) g} \sum_{i=1}^{k^{L-1}} \pi_{i}\right) .
\end{aligned}
$$

Here and bellow we assume that $\sum_{x}^{y} 1=0$ for $y<x$. So, $k^{L-1} \geq k^{L}$ can be found as follows:

(i) ${\overline{k^{L-1}}}^{L}=k^{L}$ if $\bar{T}^{L-1} \leq \varphi_{k^{L}+1}^{L-1}$,

(ii) otherwise $k^{L-1}$ is given by the condition:

$$
\varphi_{k^{L-1}}^{L-1}<\bar{T}^{L-1} \leq \varphi_{k^{L-1}+1}^{L-1},
$$

where

$$
\begin{aligned}
\varphi_{k}^{L-1} & =\sum_{i=k^{L}+1}^{k} \frac{\pi_{i}}{1+(L-2) g}\left(N_{k}^{0}-N_{i}^{0}\right) \\
& +\sum_{i=1}^{k^{L}} \frac{\pi_{i}}{1+(L-1) g} \\
& \times\left(\frac{1+(L-1) g}{1+(L-2) g} N_{k}^{0}-N_{i}^{0}-\frac{g}{1+(L-2) g} t_{*}^{L-1}\right),
\end{aligned}
$$

for $k \in\left[k^{L}+1, n\right]$ and $\varphi_{n+1}^{L-1}=\infty$.

Thus, the equilibrium strategy $T^{(L-1) *}$ of user $L-1$ is given by

$T_{i}^{(L-1) *}= \begin{cases}\frac{t_{*}^{L-1}}{1+(L-2) g} & \\ -\frac{1+(L-2) g}{1+} t_{*}^{L}+N_{i}^{0} & \\ \frac{1+(L-1) g}{1+(L-2) g}\left(t_{*}^{L-1}-N_{i}^{0}\right), & i \in\left[1, k^{L}\right], \\ 0, & i \in\left[k^{L}+1, k^{L-1}\right],\end{cases}$

Step for construction of $T^{M *}$ where $M<L$. We have already constructed $T^{L *}, \ldots, T^{(M+1) *}$ and now we are going to construct $T^{M *}$. Since $t_{*}^{M}$ is the root of the equation $\tilde{H}^{M}\left(\cdot, t_{*}^{M+1}, \ldots, t_{*}^{L}\right)=\bar{T}^{M}$ there is $k^{M}$ such that $k^{M} \geq$ $k^{M+1}$ and $N_{k^{M}+1}^{0} \geq t_{*}^{M}>N_{k^{M}}^{0}$. Thus,

$$
\begin{aligned}
t_{*}^{M} & =\left(\bar{T}^{M}+\frac{1}{1+(L-1) g} \sum_{i=1}^{k_{M}} \pi_{i}\left(A^{k, k}-N_{i}^{0}\right)\right. \\
& \left.+\sum_{r=M+1}^{L} \sum_{i=k^{p}+1}^{k^{p-1}} \frac{\pi_{i}\left(A^{p, r}-N_{i}^{0}\right)}{1+(L-1-r+p) g}\right) \\
& /\left(\frac{1}{1+(M-1) g} \sum_{i=1}^{k^{M}} \pi_{i}\right) .
\end{aligned}
$$

So, $k^{M} \geq k^{M+1}$ can be found as follows:

(i) $k^{M}=k^{M+1}$ if $\bar{T}^{M} \leq \varphi_{k^{M+1}+1}^{M}$, 
(ii) otherwise $k^{M}$ is given by the condition:

$$
\varphi_{k^{M}}^{M}<\bar{T}^{M} \leq \varphi_{k^{M}+1}^{M}
$$

where

$$
\begin{aligned}
\varphi_{k}^{M} & =\frac{1}{1+(L-1) g} \sum_{i=1}^{k} \pi_{i}\left(b^{k, k} N_{k}^{0}+A^{k, k}-N_{i}^{0}\right) \\
& +\sum_{r=M+1}^{L} \sum_{i=k^{p}+1}^{k^{p-1}} \frac{\pi_{i}\left(b^{p, r} N_{k}^{0}+A^{p, r}-N_{i}^{0}\right)}{1+(L-1-r+p) g} .
\end{aligned}
$$

Thus, the equilibrium strategy of user $M$ is given as follows

$$
T_{i}^{M *}= \begin{cases}\frac{\tau_{M}^{M}-N_{i}^{0}}{1+(L-1) g}, & i \in\left[1, k^{L}\right], \\ \frac{\tau_{r}^{M}-N_{i}^{0}}{1+(P-1) g}, & i \in\left[k^{P+1}+1, k^{P}\right], \\ 0, & P=L+M-r, r \in[M+1, L] \\ 0 & i \in\left[k^{M}+1, n\right] .\end{cases}
$$

In particular for two and three person games $(L=2$ and $L=3$ ) we have the following results.

Theorem 6: Let $\bar{T}_{1}>\bar{T}_{2}$. Then, the Nash equilibrium strategies are given by

$$
\begin{aligned}
& T_{i}^{1 *}= \begin{cases}t_{*}^{1}-\frac{g t_{*}^{2}+N_{i}^{0}}{1+g} & \text { if } i \in\left[1, k^{2}\right], \\
t_{*}^{1}-N_{i}^{0} & \text { if } i \in\left[k^{2}+1, k^{1}\right], \\
0 & \text { if } i \in\left[k^{1}+1, n\right],\end{cases} \\
& T_{i}^{2 *}= \begin{cases}\frac{1}{1+g}\left(t_{*}^{2}-N_{i}^{0}\right) & \text { if } i \in\left[1, k^{2}\right], \\
0 & \text { if } i \in\left[k^{2}+1, n\right],\end{cases}
\end{aligned}
$$

where

(a) $k^{2}, t_{*}^{2}$ are given by

$$
t_{*}^{2}=\frac{(1+g) \bar{T}^{2}+\sum_{i=1}^{k_{2}} \pi_{i} N_{i}^{0}}{\sum_{i=1}^{k_{2}} \pi_{i}},
$$

$k_{2}$ can be found from the condition

$$
\varphi_{k^{2}}^{2}<\bar{T}^{2} \leq \varphi_{k^{2}+1}^{2},
$$

where

$$
\varphi_{k}^{2}=\frac{1}{1+g} \sum_{i=1}^{k} \pi_{i}\left(N_{k}^{0}-N_{i}^{0}\right),
$$

for $k \leq n$, and $\varphi_{n+1}^{2}=\infty$,

(b) $k^{1}$ and $t_{*}^{1}$ are given by

$$
t_{*}^{1}=\frac{\bar{T}^{1}+\sum_{i=k^{2}+1}^{k^{1}} \pi_{i} N_{i}^{0}+\frac{1}{1+g} \sum_{i=1}^{k^{2}} \pi_{i}\left(g t_{2}^{*}+N_{i}^{0}\right)}{\sum_{i=1}^{k^{1}} \pi_{i}},
$$

$k^{1} \geq k^{2}$ can be found as follows:

(i) $k^{1}=k^{2}$ if $\bar{T}^{1} \leq \varphi_{k^{2}+1}^{1}$

(ii) otherwise $k^{1}$ is given by the condition:

$$
\varphi_{k^{1}}^{1}<\bar{T}^{1} \leq \varphi_{k^{1}+1}^{1}
$$

where

$$
\begin{aligned}
\varphi_{k}^{1} & =\sum_{i=k^{2}+1}^{k} \pi_{i}\left(N_{k}^{0}-N_{i}^{0}\right) \\
& +\frac{1}{1+g} \sum_{i=1}^{k^{2}} \pi_{i}\left((1+g) N_{k}^{0}-N_{i}^{0}-g t_{*}^{2}\right)
\end{aligned}
$$

for $k \in\left[k_{2}+1, n\right]$, and $\varphi_{n+1}^{1}=\infty$.

Theorem 7: Let $\bar{T}_{1}>\bar{T}_{2}>\bar{T}_{3}$. Then, the Nash equilibrium strategies are given by

$$
\begin{aligned}
& T_{i}^{1 *}= \begin{cases}t_{*}^{1}-\frac{g t_{*}^{2}}{1+g}-\frac{g t_{*}^{3}}{1+g}+N_{i}^{0} & \text { if } i \in\left[1, k^{3}\right], \\
t_{*}^{1}-\frac{g t_{*}^{2}+N_{i}^{0}}{1+g} & \text { if } i \in\left[k^{3}+1, k^{2}\right], \\
t_{*}^{1}-N_{i}^{0} & \text { if } i \in\left[k^{2}+1, k^{1}\right], \\
0 & \text { if } i \in\left[k^{1}+1, n\right],\end{cases} \\
& T_{i}^{2 *}= \begin{cases}\frac{t_{*}^{2}}{1+g}-\frac{g}{1+g} t_{*}^{3}+N_{i}^{0} & \text { if } i \in\left[1, k^{3}\right], \\
\frac{1}{1+g}\left(t_{*}^{2}-N_{i}^{0}\right) & \text { if } i \in\left[k^{3}+1, k^{2}\right], \\
0 & \text { if } i \in\left[k^{2}+1, n\right],\end{cases} \\
& T_{i}^{3 *}= \begin{cases}\frac{1}{1+2 g}\left(t_{*}^{3}-N_{i}^{0}\right) & \text { if } i \in\left[1, k^{3}\right], \\
0 & \text { if } i \in\left[k^{3}+1, n\right],\end{cases}
\end{aligned}
$$

where

(a) $k^{3}, t_{*}^{3}$ are given by

$$
\begin{gathered}
t_{*}^{3}=\left((1+2 g) \bar{T}^{3}+\sum_{i=1}^{k_{3}} \pi_{i} N_{i}^{0}\right) /\left(\sum_{i=1}^{k_{3}} \pi_{i}\right), \\
\varphi_{k^{3}}^{3}<\bar{T}^{3} \leq \varphi_{k^{3}+1}^{3}
\end{gathered}
$$

and

$$
\varphi_{k}^{3}=\frac{1}{1+2 g} \sum_{i=1}^{k} \pi_{i}\left(N_{k}^{0}-N_{i}^{0}\right),
$$

for $k \leq n$, and $\varphi_{n+1}^{3}=\infty$,

(b) $k^{2}, t_{*}^{2}$ are given by

$$
\begin{aligned}
t_{*}^{2} & =\left(\bar{T}^{2}+\frac{1}{1+g} \sum_{i=k^{3}+1}^{k^{2}} \pi_{i} N_{i}^{0}\right. \\
& \left.+\frac{1}{1+2 g} \sum_{i=1}^{k^{3}} \pi_{i}\left(\frac{g t_{*}^{3}}{1+g}+N_{i}^{0}\right)\right) /\left(\frac{1}{1+g} \sum_{i=1}^{k^{2}} \pi_{i}\right),
\end{aligned}
$$

(i) $k^{2}=k^{3}$ if $\bar{T}^{2} \leq \varphi_{k^{3}+1}^{2}$,

(ii) otherwise $k^{2}$ is given by the condition:

$$
\varphi_{k^{2}}^{2}<\bar{T}^{2} \leq \varphi_{k^{2}+1}^{2}
$$

and

$$
\begin{aligned}
\varphi_{k}^{2} & =\sum_{i=k^{3}+1}^{k} \frac{\pi_{i}}{1+g}\left(N_{k}^{0}-N_{i}^{0}\right) \\
& +\sum_{i=1}^{k^{3}} \pi_{i}\left(\frac{1}{1+g} N_{k}^{0}-\frac{N_{i}^{0}+g t_{*}^{3} /(1+g)}{1+2 g}\right) .
\end{aligned}
$$


for $k \in\left[k^{3}+1, n\right]$ and $\varphi_{n+1}^{2}=\infty$

(c) $k^{1}, t_{*}^{1}$ are given by

$$
\begin{aligned}
t_{*}^{1}= & \bar{T}^{1}+\sum_{i=k^{2}+1}^{k^{1}} \pi_{i} N_{i}^{0}+\sum_{i=k^{3}+1}^{k^{2}} \pi_{i} \frac{g t_{*}^{2}+N_{i}^{0}}{1+g} \\
& \left.+\sum_{i=1}^{k^{3}} \pi_{i}\left(\frac{g t_{*}^{2}}{1+g}+\frac{\frac{g t_{*}^{3}}{1+g}+N_{i}^{0}}{1+2 g}\right)\right) / \sum_{i=1}^{k^{1}} \pi_{i} .
\end{aligned}
$$

So, $k^{1} \geq k^{2}$ can be found as follows:

(i) $k^{1}=k^{2}$ if $\bar{T}^{1} \leq \varphi_{k^{2}+1}^{1}$,

(ii) otherwise $k^{1}$ is given by the condition:

$$
\varphi_{k^{1}}^{1}<\bar{T}^{1} \leq \varphi_{k^{1}+1}^{1}
$$

where

$$
\begin{gathered}
\varphi_{k}^{1}=\sum_{i=k^{2}+1}^{k} \pi_{i}\left(N_{k}^{0}-N_{i}^{0}\right)+\sum_{i=k^{3}+1}^{k^{2}} \pi_{i}\left(N_{k}^{0}-\frac{g t_{*}^{2}+N_{i}^{0}}{1+g}\right) \\
+\sum_{i=1}^{k^{3}} \pi_{i}\left(N_{k}^{0}-\frac{g t_{*}^{2}}{1+g}-\frac{\frac{g t_{*}^{3}}{1+g}+N_{i}^{0}}{1+2 g}\right) . \\
\text { VIII. NUMERICAL EXAMPLES }
\end{gathered}
$$

Let us demonstrate the closed form approach by numerical examples. Take $n=5, N_{i}^{0}=\kappa^{i-1}, \kappa=1.7, \pi_{i}=1 / 5$ for $i \in[1,5]$. We consider the cases 1,2 and 3 users scenari.

Single user scenario. Let $\bar{T}=5$. Then, by Theorem 2 as the first step we calculate $\varphi_{t}$ for $t \in[1,5]$. In our case we get $(0,0.14,0.616,1.830,4.581)$. Thus, we have $k=5$ and the optimal water-filling strategy is $T^{*}=$ $(7.771,7.071,5.881,3.858,0.419)$ with payoff 1.11 .

Two users scenario. Let also $g=0.9, \bar{T}^{1}=5, \bar{T}^{2}=1$. Then, by Theorem 6 as the first step we calculate $\varphi_{t}^{2}$ for $t \in[1,5]$. In our case we get $(0,0.074,0.324,0.963,2.411)$. Thus, $k^{2}=4$ and $t_{*}^{2}=5.001$. Then we calculate $\varphi_{t}^{1}$ for $t=5$. In our case we get 6.994. Thus, $k^{1}=4$ and $t_{*}^{1}=0.010$. Therefore, we have the following equilibrium strategies $T^{1 *}=(7.106,6.737,6.111,5.046,0)$ and $T^{2 *}=$ $(2.106,1.737,1,111,0.0462,0)$ with payoffs 0.801 and 0.116 , respectively.

Three users scenario. Let us introduce the third player with the average power constraint $\bar{T}^{3}=0.5$. Then, by Theorem 7 we can find that $T^{1 *}=(6.419,6.169,5.744,4.900,1.769)$, $T^{2 *}=(1.861,1.611,1.186,0.342,0)$ and $T^{3 *}=$ $(1.142,0.892,0.467,0,0)$ are equilibrium strategies with payoffs $0.728,0.113$ and 0.055 , respectively.

The equilibrium strategies of all three cases are shown in Figure 1. When a new user comes into competition, it leads to a bigger rivalry for using good quality channels and it results in the situation when bad quality channels turn out to become more attractive for users than they were when there were smaller number of users.

We have run IWFA, which produced the same values for the equilibrium strategies and payoffs. However, we have observed

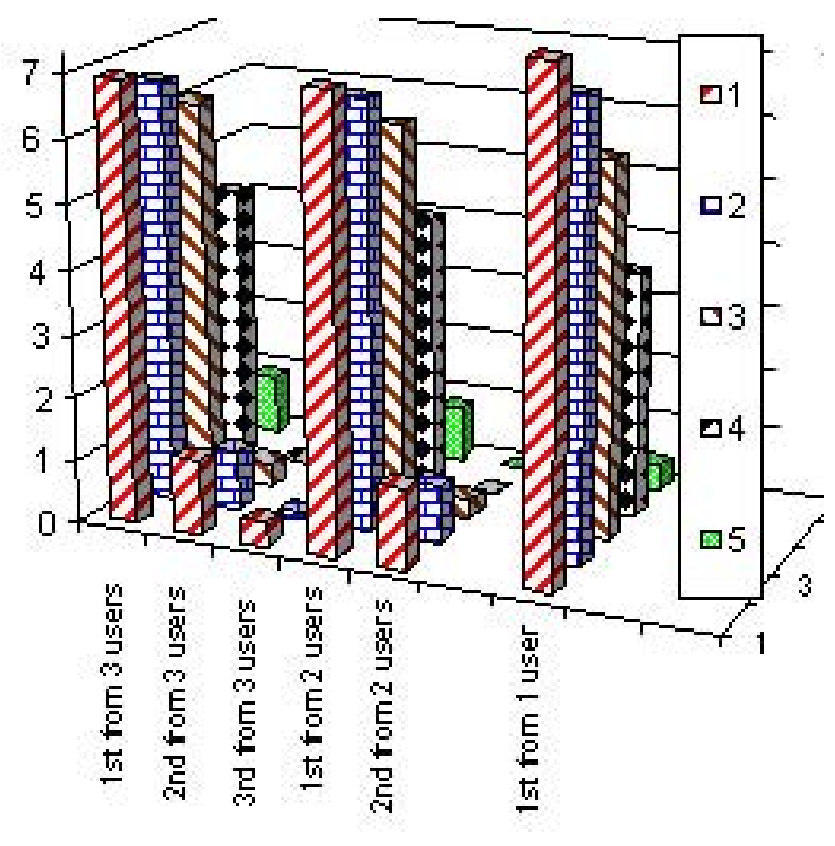

Fig. 1. Optimal strategies for 1,2 and 3 user games

that the convergence of IWFA is slow when $g \approx 1$. In Figure 2 , for the two users scenario, we have plotted the total error in strategies $\left\|T_{k}^{1}-T^{1 *}\right\|_{2}+\left\|T_{k}^{2}-T^{2 *}\right\|_{2}$, where $T_{k}^{i}$ are the strategies produced by IWFA on the $k$-th iteration and $T^{i *}$ are the Nash equilibrium strategies. Our approach instantaneously finds the Nash equilibrium for all values of $g$. Also, it is interesting to note that by Theorems 6 and 7 the quantity of channels as well as the channels themselves used by weaker user (with smaller resources) is independent from the behavior of the stronger user (with larger resources). Of course, each user allocates his/her resources among the channels taking into account the opponent behavior.

In Figures 3 and 4, we compare the non-cooperative approach with the cooperative approach. Specifically, we compare the transmission rates and their sum under Nash equilibrium strategies and under strategies obtained from the centralized optimization of the sum of users' rates. The main conclusions are: the cost of anarchy is nearly zero for $g \in$ $[0,1 / 4]$ and then it grows up to $22 \%$ when $g$ grows from $1 / 4$ to 1 ; the user with more resources gains significantly more from the centralized optimization. Hence, the non-cooperative approach results in a more fair resource distribution. In Figure 4 we plot the total transmission rate under Nash equilibrium strategies and under strategies obtained from the centralized optimization for the cases of 2 and 3 users. As expected the introduction of a new user increases the cost of anarchy. Furthermore, in the case of the centralized optimization with the introduction of a new user the total rate increases, and on contrary in the game setting the total rate decreases.

\section{Conclusion}

We have considered power control for wireless networks in optimization and game frameworks. Closed form solutions for 


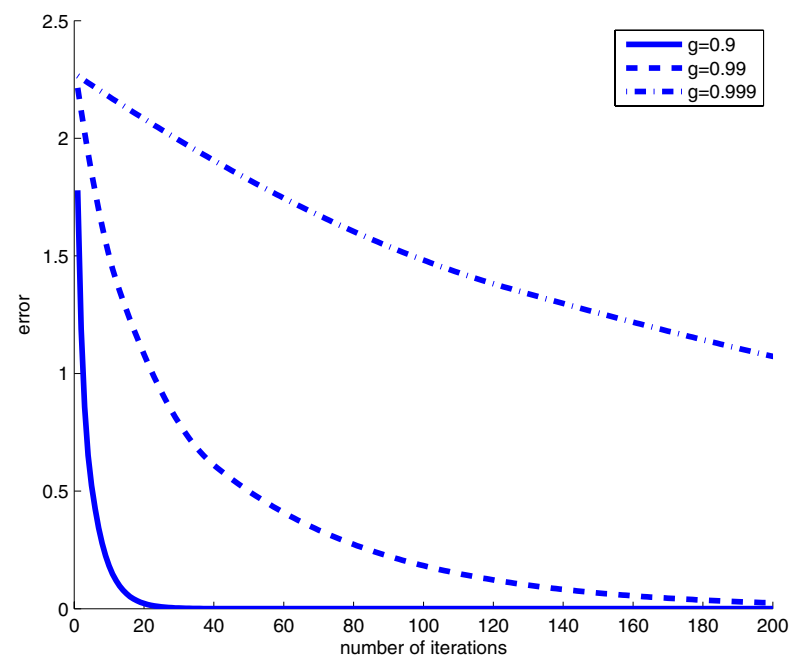

Fig. 2. Convergence of IWFA

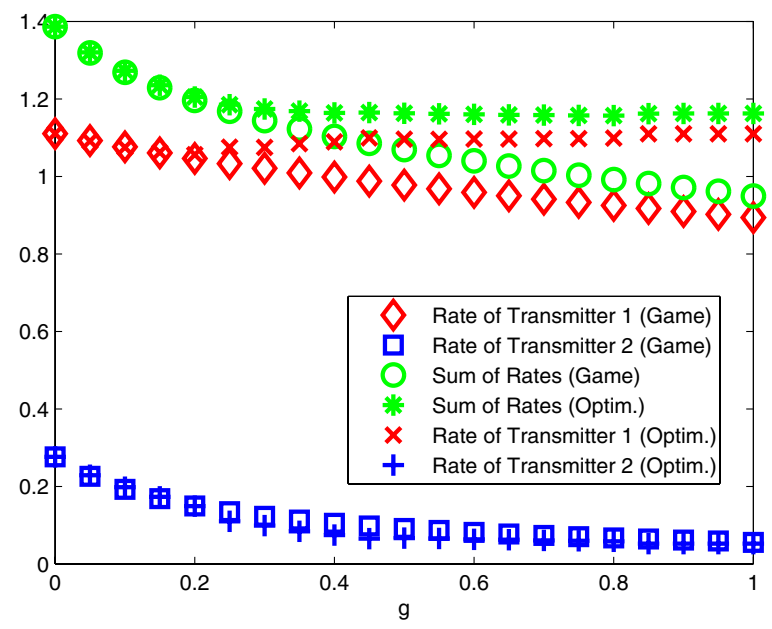

Fig. 3. Centralized Optimization vs. Game

the water filling optimization problem and $L$ users symmetric water filling games have been provided. Namely, now one can calculate equilibrium strategies with a finite number of arithmetic operations. This was possible due to the intrinsic hierarchical structure induced by the quantity of the resources available to the users. We have also provided a simple alternative proof of convergence for a version of iterative water filling algorithm. It had been known before that the iterative water filling algorithm converges very slow when the crosstalk coefficient is close to one. For our closed form approach possible proximity of the crosstalk coefficient to one is not a problem. We have shown that when the crosstalk coefficient is equal to one, there is a continuum of NE. Finally, we have demonstrated that the price of anarchy is small when the crosstalk coefficient is small and that the decentralized solution is better than the centralized one with respect to fairness.

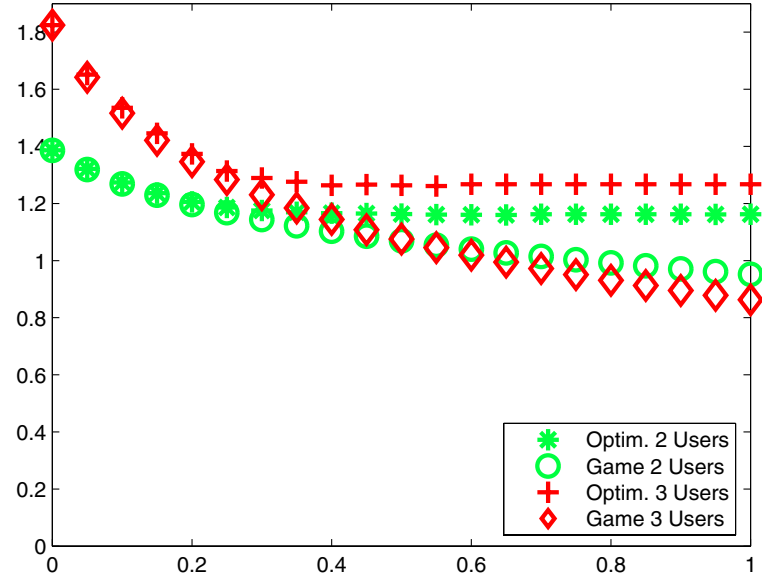

Fig. 4. The effect of a new user

\section{REFERENCES}

[1] E. Altman, K. Avrachenkov, A. Garnaev, "A jamming game in wireless networks with transmission cost". in Proc. of NET-COOP 2007. Lecture Notes in Computer Science, v.4465, pp.1-12, 2007.

[2] E. Altman, K. Avrachenkov, G. Miller and B. Prabhu, "Discrete power control: cooperative and non-cooperative optimization", in Proceedings of IEEE INFOCOM 2007. An extended version is available as INRIA Research Report no.5818.

[3] T. Cover and J. Thomas, Elements of Information Theory, Wiley, 1991.

[4] W. R. Heinzelman, A. Chandrakasan, and H. Balakrishnan, "Energyefficient communication protocol for wireless microsensor networks," in Proc. of the 33rd Annual Hawaii International Conference on System Sciences, v.2, Jan. 2000.

[5] A. Garnaev, Search Games and Other Applications of Game Theory, Springer, 2000.

[6] A.J. Goldsmith and P.P. Varaiya, "Capacity of fading channels with channel side information", IEEE Trans. Information Theory, v.43(6), pp.1986-1992, 1997.

[7] T. J. Kwon and M. Gerla, "Clustering with power control," in Proc. IEEE Military Communications Conference (MILCOM'99), v.2, Atlantic City, NJ, USA, 1999, pp.1424-1428.

[8] L. Lai and H. El Gamal, "Fading Multiple Access Channels: A Game Theoretic Perspective", IEEE International Symposium on Information Theory (ISIT), Seattle, WA, July 2006, pp.1334-1338.

[9] C. R. Lin and M. Gerla, "Adaptive clustering for mobile wireless networks," IEEE JSAC, v.15, no.7, pp.1265-1275, 1997.

[10] Z.-Q. Luo and J.-S. Pang, "Analysis of iterative waterfilling algorithm for multiuser power control in digital subscriber lines", EURASIP Journal on Applied Signal Processing, v.2006, 10 pages, 2006.

[11] O. Popescu and C. Rose, "Water filling may not good neighbors make", in Proceedings of GLOBECOM 2003, v.3, pp.1766-1770, 2003.

[12] D.C. Popescu, O. Popescu and C. Rose, "Interference avoidance versus iterative water filling in multiaccess vector channels", in Proceedings of IEEE VTC 2004 Fall, v.3, pp.2058-2062, 2004.

[13] K.B. Song, S.T. Chung, G. Ginis and J.M. Cioffi, "Dynamic spectrum management for next-generation DSL systems, IEEE Communications Magazine, v.40, pp.101-109, 2002.

[14] D. Tse and P. Viswanath, Fundamentals of Wireless Communication, Cambridge University Press, 2005.

[15] W. Yu, Competition and cooperation in multi-user communication environements, PhD Thesis, Stanford University, June 2002.

[16] W. Yu, G. Ginis and J.M. Cioffi, "Distributed multiuser power control for digital subscriber lines", IEEE JSAC, v.20, pp.1105-1115, 2002. 